\title{
Monopole Antenna With Inkjet-Printed EBG Array on Paper Substrate for Wearable Applications
}

\author{
Sangkil Kim, Student Member, IEEE, Yu-Jiun Ren, Member, IEEE, Hoseon Lee, Amin Rida, Member, IEEE, \\ Symeon Nikolaou, Member, IEEE, and Manos M. Tentzeris, Fellow, IEEE
}

\begin{abstract}
In this letter, a novel electromagnetic band-gap structure (EBG) with single-ring resonators is inkjet-printed on the commercially available photo paper using conductive nano-silver ink. The printed EBG array is placed above a copper sheet, forming an artificial magnetic conductor (AMC) reflector at the designed frequency range $(2.4 \sim 2.5 \mathrm{GHz})$. A microstrip monopole antenna is backed with the designed $A M C$ reflector and is tested in free space and in contact with a human phantom. The antenna gain of a conventional microstrip monopole on human phantom is as low as $-9 \mathrm{dBi}$. The gain of the proposed AMC backed monopole, measured on a human phantom is $0.95 \mathrm{dBi}$. The measurements demonstrate superior performance of the proposed monopole with EBG array compared to a conventional microstrip monopole antenna when they are considered for wearable applications.
\end{abstract}

Index Terms-Artificial magnetic conductor (AMC), electromagnetic band-gap (EBG) structure, inkjet printing, personal area networks (PANs), wearable antenna, wireless body area networks (WBANs).

\section{INTRODUCTION}

$\mathbf{U}$ TILIZATION of a wearable radio system is becoming more and more important because of the constantly increasing demand for tracking, navigation, mobile computing and public safety applications. IEEE 802.15 standard has been established to standardize applications for personal area networks (PANs) and body area networks (BANs). One of the major challenges for the implementation of wearable radio systems is the degrading effect of the human body [1], [2] The human body is a very lossy, dispersive material with high dielectric constant $\left(\varepsilon_{\mathrm{r}} \approx 35\right)$ that absorbs a large amount of the radiated electromagnetic power from the antenna. Therefore, it is very common for the gain of the antennas to significantly decrease when the antennas operate close to a human body, which leads to deterioration of the communication range of the radio system as well. In addition, the body-absorbed electromagnetic power may cause unwanted, adverse biological effects. To overcome these challenges, electromagnetic band-gap structures (EBGs) have been suggested to isolate the antenna from the ambient environment [3]-[6].

Manuscript received March 14, 2012; revised May 18, 2012; accepted May 28, 2012. Date of publication June 06, 2012; date of current version June 22, 2012. This work was supported by Semiconductor Research Corporation/Interconnect Focus Center (SRC/IFC) and Cyprus' Research Promotion Foundation under Program PENEK/ENISX/0308/72.

S. Kim, H. Lee, A. Rida, and M. M. Tentzeris are with the Georgia Institute of Technology, Atlanta, GA 30332 USA (e-mail: ksangkil3@gatech.edu).

Y.-J. Ren is with Research In Motion Corporation, Dallas, TX 75039 USA (e-mail: yjren03@gmail.com).

S. Nikolaou is with the Electrical Engineering Department, Frederick University, Nicosia 1036, Cyprus (e-mail:simos.nikolaou@gmail.com).

Color versions of one or more of the figures in this letter are available online at http://ieeexplore.ieee.org.

Digital Object Identifier 10.1109/LAWP.2012.2203291

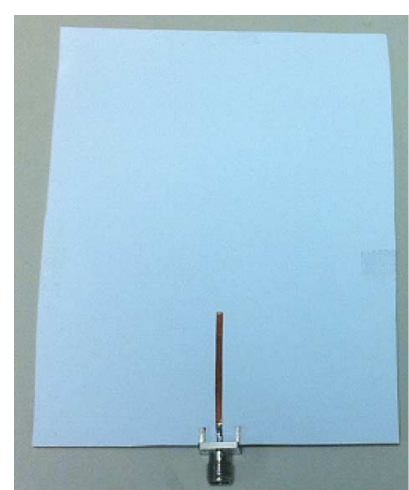

(a)

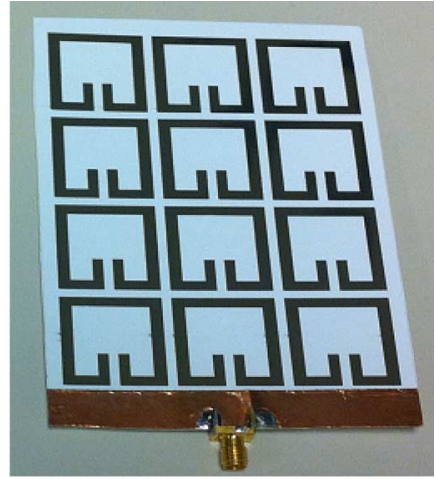

(b)
Fig. 1. Fabricated antenna. (a) Front view. (b) Rear view.

In this letter, a microstrip monopole antenna, backed with an inkjet-printed EBG array and a metallic copper sheet, is proposed. The combination of an EBG array and a metal layer effectively forms an artificial magnetic conductor (AMC) reflector at the designed frequency band of $2.4 \sim 2.5 \mathrm{GHz}$, and consequently isolates the radio system from the human body effect, alleviating the common on-body antenna performance degradation. In this letter, an EBG structure was implemented using a $4 \times 3$ array of single split-ring resonators [7]. The EBG array was fabricated using inkjet-printing technology on the commercially available photo paper, which is a low-cost, flexible, recyclable material. The area of the proposed EBGs' unit cell is $60 \%$ smaller and one-third thick, compared to previous designs reported in [8] and [6], respectively.

\section{ANTENNA ARCHITECTURE}

\section{A. Geometry}

The proposed structure consists of a microstrip linear monopole, backed with an EBG array (4 rows by 3 columns) inkjet-printed on the same side with the RF ground plane. Commercially available 0.23 -mm-thick paper is used as the RF substrate, with dimensions $127 \times 87 \mathrm{~mm}^{2}$. The fabricated EBG-backed microstrip monopole can be seen in Fig. 1. On the front side of the paper substrate [Fig. 1(a)], the linear monopole antenna is $37.4 \mathrm{~mm}$ long and $1.3 \mathrm{~mm}$ wide, resulting in a $50-\Omega$, characteristic impedance $\left(Z_{\mathrm{o}}\right)$. An RF copper ground plane with dimensions $10 \times 87 \mathrm{~mm}^{2}$ is attached on the rear side of the paper substrate [Fig. 1(b)]. On the same side, a $4 \times 3$ array of EBG cells is inkjet-printed. The array of EBG cells is placed above a metallic layer that forms an artificial magnetic conductor (AMC). The AMC is used to improve the performance of the antenna when it is placed on the human body for a wearable application. 


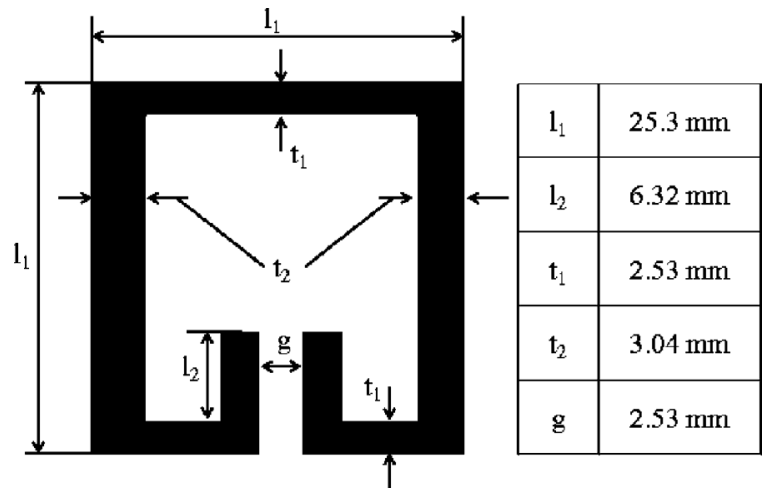

Fig. 2. Unit cell of EBG structure. The distance between each cell is $2.53 \mathrm{~mm}$.

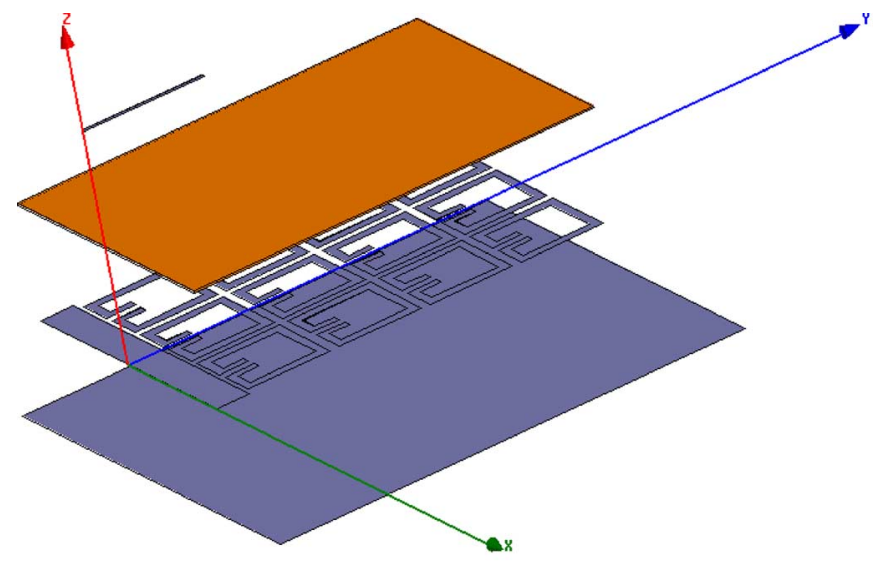

(a)

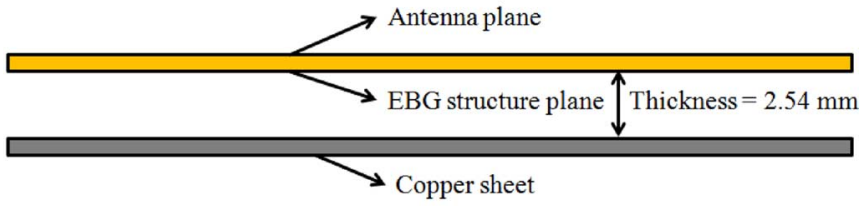

(b)

Fig. 3. (a) Antenna geometry (b) Antenna profile. The thickness of the paper substrate is $0.46 \mathrm{~mm}$, and the copper sheet is $0.1 \mathrm{~mm}$.

The dimensions and the exact shape of each EBG unit cell are summarized in Fig. 2. The distance between adjacent cells is $2.53 \mathrm{~mm}$. The distance between the bottom row and the copper RF ground segment is $1.27 \mathrm{~mm}$. A 0.1 -mm-thick copper sheet with dimensions $150 \times 120 \mathrm{~mm}^{2}$ is used as the metallic reflector, and a 2.54-mm-thick Styrofoam layer is inserted as spacer between the EBG array and the metallic reflector. In Fig. 3, the EBG-backed microstrip monopole is placed on top of the Styrofoam slab, and the Styrofoam slab is placed on top of the metallic reflector.

\section{B. Fabrication Process}

First of all, the EBG structure is printed by inkjet-printing technology. A commercially available drop-on-demand inkjet printer is used to print EBG structure on paper substrate. The properties of the paper substrate were studied through the use of the microstrip ring resonators [9]. The dielectric constant $\left(\varepsilon_{\mathrm{r}}\right)$ of the paper substrate that is $0.23 \mathrm{~mm}$ thick is about 3.2 at $2.4 \sim 2.5-\mathrm{GHz}$ band. The loss tangent $(\tan \delta)$ is about 0.07 throughout the frequency band. For printing, Dimatix $10 \mathrm{pL}$ cartridge was utilized, and it was kept at a distance of $800 \mu \mathrm{m}$ from the surface of the paper on a printing plate of the printer. The printer-head angle was adjusted to achieve a print resolution of 1270 dpi. Cabot conductive ink CCI-300 was jetted at the temperature of $36^{\circ} \mathrm{C}$, while the paper substrate was maintained at $50^{\circ} \mathrm{C}$. The printed pattern was sintered in a thermal oven for $2 \mathrm{~h}$ at $130^{\circ} \mathrm{C}$.

Next, a monopole antenna and ground plane for antenna feeding is cut out from copper tape and attached on the substrate. The monopole antenna is located on the center of the substrate. Commercial Styrofoam foam, whose dielectric constant $\left(\varepsilon_{\mathrm{r}}\right)$ is close to that of air, is inserted between the paper substrate and the copper sheet.

\section{AMC REFLeCtor Using EBG StRUCTURE}

It has been shown that an EBG structure placed above a metallic layer (AMC reflector) can improve the antenna performance when the antenna operates adjacently to the human body [8]. Therefore, an AMC reflector using EBG structure is designed to isolate the performance of the microstrip monopole antenna, designed for wearable applications, from the detrimental effect of a human body. The AMC reflector is designed by means of reflection phase characterization [10], [11]. First, an infinite EBG structure is placed above the metallic layer. Then, a plane wave is illuminated onto the infinite EBG surface, and the phase of reflection coefficient $\left(S_{11}\right)$ on the surface of the $\mathrm{AMC}$ reflector is monitored. Linearly polarized plane waves are used because the EBG surface is composed of polar-sensitive open ring operates, which resonates in quasi-static mode. The surface of the EBG structure can be considered as an AMC reflector if the reflection phase from the surface is in the range of $-90^{\circ} \sim+90^{\circ}$ since the reflected waves will be constructively added to radiated waves [11].

The detailed dimensions of a unit cell of the designed EBG structure are described in Fig. 2. The overall size of the ring is $25.3 \times 25.3 \mathrm{~mm}^{2}$ with gap $(\mathrm{g})$ of $2.53 \mathrm{~mm}$. The thicknesses of the top and bottom sides $\left(t_{1}\right)$ are $2.53 \mathrm{~mm}$, and left and right sides $\left(t_{2}\right)$ are $3.04 \mathrm{~mm}$, respectively. Pin length $\left(l_{2}\right)$ is $6.32 \mathrm{~mm}$, while its thickness is the same as $t_{1}$. The circumference of the ring resonator corresponds to one wavelength $\left(\lambda_{0}\right)$ at the central frequency $(2.45 \mathrm{GHz})$, in free space, and the thickness of each side is adjusted to optimize the resonance frequency at $2.45 \mathrm{GHz}$. The distance between pin-to-pin $(g)$ and the cell-to-cell is selected to the same as the thickness of the top and bottom sides $\left(t_{1}\right)$ for the design simplification. For the proposed design, the distance between the EBG structure and the copper sheet is chosen according to the thickness of the commercially available Styrofoam slabs.

Two slabs of the Styrofoam are inserted to keep the distance constant. Fig. 4 shows the calculated phase of the return loss $\left(S_{11}\right)$ on the surface of an infinite AMC reflector. The $x$ - and $y$-axis polarized plane waves are illuminated in the angle of $0^{\circ}$ and $45^{\circ}$ on the AMC reflector to investigate its polarization properties. The $y$-axis polarized plane waves show in-phase responses at $2.45 \mathrm{GHz}$, while $x$-axis polarized ones have out-ofphase responses. Therefore, the radiated waves from the antenna that have a cross polarization will be suppressed by the $\mathrm{AMC}$ reflector. For the $y$-polarized wave, the reflection phase is $0^{\circ}$ at $2.45 \mathrm{GHz}$, and the range of having reflection phase from 


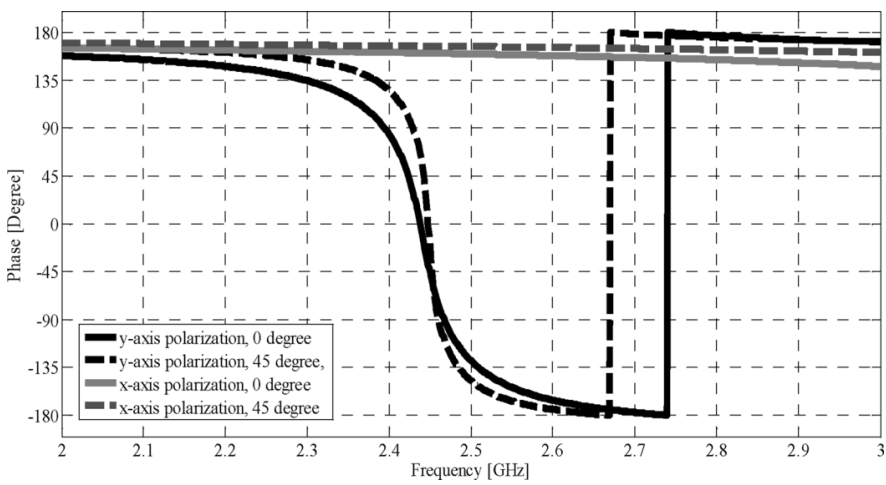

Fig. 4. Calculated band-gap response of AMC reflector.

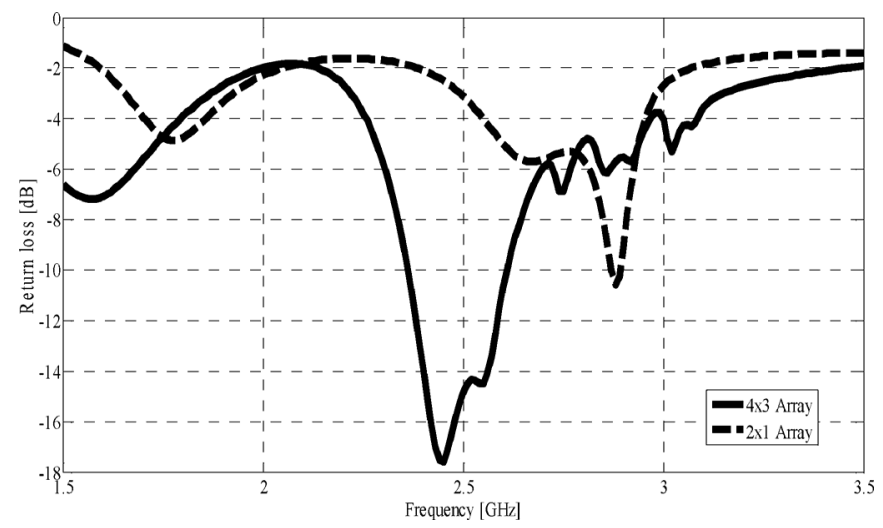

Fig. 5. Array size and return loss $\left(S_{11}\right)$ variation.

$-90^{\circ} \sim 90^{\circ}$ is $2.4 \sim 2.48 \mathrm{GHz}$. Within this frequency range, reflected wave makes constructive interference with radiated wave in the free space. However the practical array size needs to be determined since it is impossible to realize the simulated infinite array. A small size is generally desirable, however the resonating frequency of the microstrip antenna/AMC reflector changes, and the antenna does not operate adequately when the EBG array is too small. This is because the parasitic capacitance between the antenna and the EBG array affects the performance of the whole structure.

In Fig. 5, simulated return loss $\left(S_{11}\right)$ of the antenna having different EBG array size is presented in order to show the effect of the size of the EBG array. The antenna with a $2 \times 1$ EBG array does not cover the desired frequency range of 2.4 2.5 GHz. Additionally, the antenna has very poor gain due to mismatch at the desired frequency range. The array size is increased by one row and one column at a time until satisfactory performance is achieved. Finally, the fabricated $4 \times 3$ array was chosen as a compensation between relatively small size and high gain, at 2.40 2.48 GHz. In this size, the $4 \times 3$ EBG array structure above the copper sheet forms a design that approximates an AMC reflector (with ideally infinite size). Finally, the size of the copper sheet needs to be determined. A width of $20 \mathrm{~mm}$ of the copper sheet is extended from each side of antenna in order to block the backscattered waves more effectively, resulting in the copper sheet overall dimensions of $150 \times 130 \mathrm{~mm}^{2}$. In Section IV, the effective performance of the $\mathrm{AMC}$ reflector is demonstrated in the return loss measurement in Fig. 7, and the gain measurement is shown in Fig. 9.

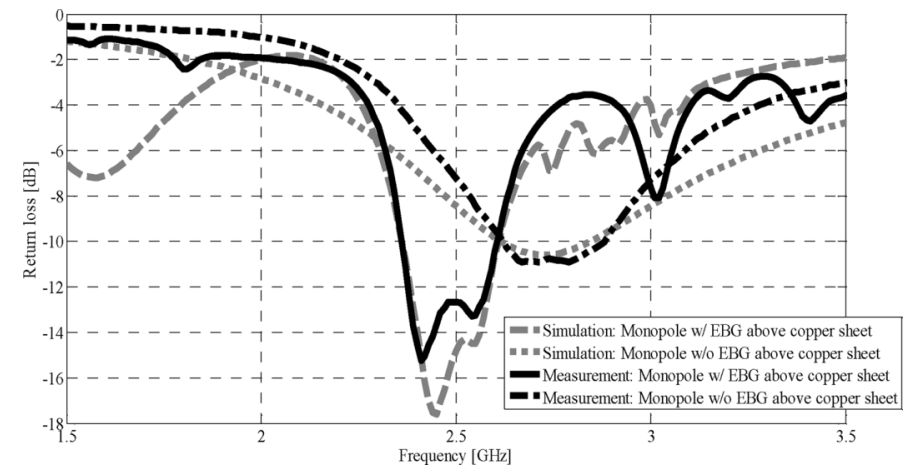

Fig. 6. Return loss $\left(S_{11}\right)$ of a microstrip monopole antenna above copper sheet with/without EBG structure.

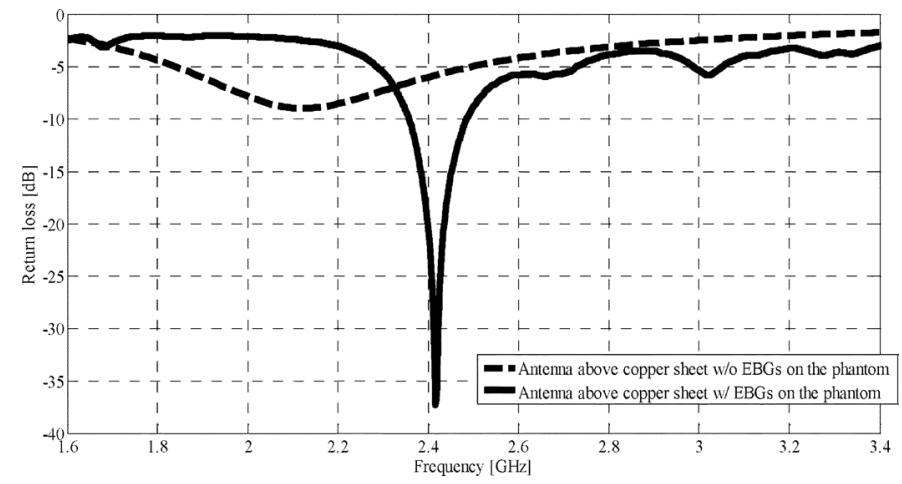

Fig. 7. Return loss $\left(S_{11}\right)$ of the antenna above copper sheet on the phantom with/without EBGs.

\section{EXPERIMENTAL RESULTS}

\section{A. Return Loss Measurement}

Measured and simulated return loss $\left(S_{11}\right)$ of the fabricated antenna is presented in Fig. 6. The effectiveness and usefulness of the EBG array can be assessed by comparing the $S_{11}$ of the antenna with (w/ EBG) and without (w/o EBG) EBG. While the proposed antenna with the AMC reflector is well matched $\left(S_{11}<-10 \mathrm{~dB}\right)$ from 2.36 to $2.61 \mathrm{GHz}$, in the absence of the EBG array, the antenna does not resonate. The return loss measurements presented in Fig. 6 have been taken inside an anechoic chamber, and the measurements are in very good agreement with the simulated results. The relatively wide fractional bandwidth $(10.06 \%)$ is a result of the designed distance between EBG array plane and the metallic sheet. The two antennas were also tested when they were attached on a human body phantom. Measurement of return loss for the microstrip monopole with and without the EBG array presented in Fig. 7 clearly indicates that the antenna does not resonate without the EBG array, demonstrating the necessity of the AMC in order for the antenna to be used for wearable applications. Return loss measurements in combination with the radiation pattern and gain measurements show that the antenna with the AMC reflector effectively reduces the effect of human body. The phantom is shaped like a human torso [12], and the antenna is placed in the middle of the phantom's chest during the measurement. The dielectric constant $\left(\varepsilon_{\mathrm{r}}\right)$ and conductivity $(\sigma)$ of the phantom are 35.4 and $1.82 \mathrm{~S} / \mathrm{m}$, respectively. 


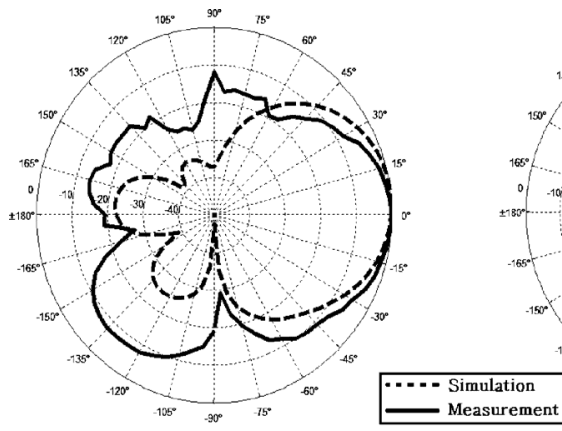

(a)

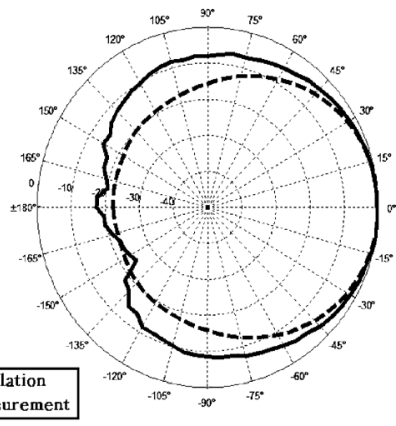

(b)
Fig. 8. Copolarized $\left(\mathrm{E}_{\varphi}\right)$ radiation pattern. (a) $y z$-plane (E-plane). (b) $x z$-plane (H-plane).

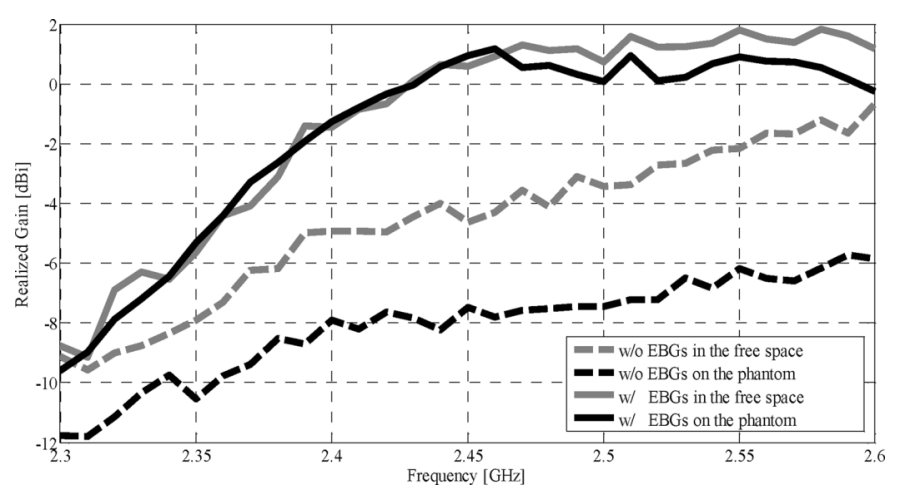

Fig. 9. Gain variation according to frequency.

\section{B. Radiation Pattern and Gain Measurement}

Radiation pattern and gain of the antenna is measured in order to evaluate the radiation properties of the antenna in free space and on the phantom. Fig. 8 presents the measured and simulated radiation patterns for the antenna in free space. $\mathrm{E}_{\varphi}$-component at $2.45 \mathrm{GHz}$ is plotted on the $y z$ - and $x z$-plane, respectively. The $y$-axis corresponds to $\varphi=0^{\circ}$ [Fig. 8(a)], and the $z$-axis corresponds to $\theta=0^{\circ}$ [Fig. 8(b)]. Simulation and measurements are evidently in good agreement. The shape of the radiation pattern is fairly consistent in the whole frequency band from 2.4 to $2.5 \mathrm{GHz}$ and is almost the same with the presented pattern at $2.45 \mathrm{GHz}$. To confirm the superior performance of the monopole with the EBG array compared to the antenna with only the metallic layer, gain measurements were taken first for the antennas in free space and, second, attached on the chest of the phantom. Regarding the antenna above copper sheet without EBGs, the distance between the monopole and the copper sheet is kept the same as the antenna above the copper sheet with EBGs in order to demonstrate the effect of the EBGs only.

A few important observations can be made from the measurements presented in Fig. 9. The first is that the phantom deteriorates the gain of the antenna when the AMC is not implemented. At $2.45 \mathrm{GHz}$, the simulated gain of a conventional monopole antenna on the phantom without EBGs or ground plane is -9.38 $\mathrm{dBi}$, while the measured gain of the antenna with the metal sheet on the phantom is $-7.49 \mathrm{dBi}$, which is about $2.8 \mathrm{dBi}$ lower than that of the antenna in the free space. The second is that EBG array and the resulted $\mathrm{AMC}$ increases the gain to higher than $0 \mathrm{dBi}$, and the third is that AMC effectively isolates the antenna from the backside environment since the gain remains the same in both the presence and the absence of the phantom. When the gain of the proposed antenna is measured on the phantom, it presents a more than $8-\mathrm{dBi}$ increase compared to the same antenna without the EBG array, proving the usefulness of the resulted AMC, for wearable applications. These results are in agreement with the simulation results since the simulated gain of the antenna with metal sheet on the phantom, without EBGs, at $2.45 \mathrm{GHz}$ is $-8.12 \mathrm{dBi}$, and the gain of the monopole with $\mathrm{AMC}$ is $0.86 \mathrm{dBi}$. Therefore, the detrimental effect of lossy materials like the human body on the antenna gain is very small due to the AMC reflector, which can allow the increase of the maximum communication range.

\section{CONCLUSION}

In this letter, a microstrip monopole antenna with an inkjet-printed EBG array is proposed for wearable applications. The proposed antenna with the integrated AMC reflector has very low profile $\left(0.025 \lambda_{2.45}\right)$ and improved gain. Free-space and on-phantom measurements for $S_{11}$, radiation pattern, and gain were conducted to verify the antenna's performance. The measured $-10-\mathrm{dB}$ bandwidth covers the whole frequency range of the IEEE 802.15 standard. At the same time, the reported gain increase of $8.44 \mathrm{dBi}$ for the antenna on phantom indicates that the monopole antenna with EBG array is a very good candidate for wearable electronics applications.

\section{ACKNOWLEDGMENT}

The authors would like to thank the reviewers for their careful and in-depth evaluation.

\section{REFERENCES}

[1] N. H. M. Rais, P. J. Soh, F. Malek, S. Ahmad, N. B. M. Hashim, and P. S. Hall, "A review of wearable antenna," in Proc. LAPC, Loughborough, U.K., 2009, pp. 225-228.

[2] P. S. Hall and Y. Hao, "Antennas and propagation for body centric communications," in Proc. EuCAP, 2006, pp. 1-7.

[3] H. Nakano, K. Kikkawa, N. Kondo, Y. Iitsuka, and J. Yamauchi, "Lowprofile equiangular spiral antenna backed by an EBG reflector," IEEE Trans. Antennas Propag., vol. 57, no. 5, pp. 1309-1318, May 2009.

[4] Y. Huang, A. De, Y. Zhang, T. K. Sarkar, and J. Carlo, "Design of a low profile end-fire antenna using split-ring resonators," in Proc. IEEE AP-S Int. Symp., 2008, pp. 1-4.

[5] Y. H. Liu and X. P. Zhao, "Investigation of anisotropic negative permeability medium cover for patch antenna," Microw., Antennas Propag., vol. 7, no. 7, pp. 737-744, 2008.

[6] E. Y. Kim, J. H. Yoon, Y. J. Yoon, and C. G. Kim, "Low profile dualband reflector antenna with dual resonant AMC," in Proc. IEEE APSURSI, 2011, pp. 1800-1803.

[7] M. Tang, S. Xiao, T. Deng, and B. Wang, "Novel folded single split ring resonator and its application to eliminate scan blindness in infinite phased array," in Proc. ISSSE, 2010, vol. 1, pp. 1-4.

[8] S. Zhu and R. Langley, "Dual-band wearable textile antenna on an EBG substrate," IEEE Trans. Antennas Propag., vol. 57, no. 4, pp. 926-934, Apr. 2009.

[9] A. Rida, L. Yang, R. Vyas, and M. M. Tentzeris, "Conductive inkjetprinted antennas on flexible low-cost paper-based substrates for RFID and WSN applications," IEEE Antennas Propag. Mag., vol. 51, no. 3, pp. 13-23, Jun. 2009.

[10] D. Sievenpiper, L. Zhang, R. F. J. Broas, N. G. Alexopoulos, and E. Yablonovich, "High-impedance electromagnetic surfaces with a forbidden frequency band," IEEE Trans. Microw. Theory Tech., vol. 47, no. 11, pp. 2059-2074, Nov. 1999.

[11] F. Yang and Y. Rahmat-Samii, "Reflection phase characterization of the EBG ground plane for low profile wire antenna applications," IEEE Trans. Antennas Propag., vol. 51, no. 10, pp. 2691-2703, Oct. 2003.

[12] MCL Technology, Ltd., Dingstow, U.K., "MCL-T whole-body solid SAM phantom," 2012 [Online]. Available: http://www.mcluk.org/ solid_bodies.php 